\title{
Diabetes tipo 1: el pediatra y los nuevos tratamientos
}

I. González Casado

Pediatra. Servicio de Endocrinología Pediátrica. Hospital Universitario La Paz. Madrid. España.

Rev Pediatr Aten Primaria. 2009; / Supl 16:s205-s215

Isabel González Casado, igonzalezc.hulp@salud.madrid.org

\section{Resumen}

La mejora del control glucémico en los pacientes con diabetes mellitus tipo 1 reduce el riesgo de complicaciones vasculares a largo plazo, incluso en la edad pediátrica, sobre todo cuando se realiza un tratamiento intensivo con múltiples dosis de insulina o con sistemas de infusión subcutánea continua de insulina (bombas). A cualquier edad el objetivo debe ser realizar una sustitución insulínica lo más fisiológica posible, intentando conseguir controles glucémicos óptimos. Sin embargo, las insulinas tradicionales presentan ciertas limitaciones clínicas que han llevado al desarrollo de nuevos análogos de insulina, de acción rápida y prolongada, que han permitido cierta mejoría en el cuidado de la diabetes.

Palabras claves: Diabetes mellitus tipo 1, Múltiples dosis de insulina, Sistemas de infusión subcutánea continua de insulina, Análogos de acción rápida, Análogos de acción prolongada.

Abstract

Improvements in glycemic control, particularly when provided by intensive insulin treatment with multiple daily inyections or pump therapy, reduce the risks of vascular complications, also in younger children. In all age-groups, as close to physiological insulin replacement as possible and optimal glycemic control must be the aim. But the fact that the traditional insulins have certain clinical limitations has led to the development of new analogues, rapid and long acting. These insulins represent some improvement in the care of diabetes.

Key words: Type 1 diabetes mellitus, Multiple daily injections, Continuous subcutaneous insulin infusion, Rapid-acting insulin analogues, Basal insulin analogues.

\section{Introducción}

La diabetes mellitus tipo 1 (DM1) se caracteriza por el déficit absoluto de insulina secundario a la destrucción autoinmune de las células $\beta$ pancreáticas.
Un buen control glucémico mediante una insulinoterapia intensiva y un adecuado autocontrol, puede reducir la incidencia de complicaciones a largo plazo y retrasar la progresión de las ya

La autora declara no presentar conflictos de intereses en relación con la preparación y publicación de este artículo. 
existentes, incluso en la población pediátrica.

El objetivo del tratamiento insulínico en la DM1 es conseguir un adecuado control metabólico, obteniendo glucemias lo más parecidas a la normalidad. Con esto se intenta prevenir o retrasar la aparición de complicaciones crónicas, disminuir las complicaciones agudas, sobre todo las hipoglucemias, y conseguir una buena calidad de vida. Sin embargo, el control metabólico de un niño con DM1 casi nunca se acerca al estado de euglucemia de un sujeto sano: la mayoría presentan hiperglucemias a pesar del tratamiento insulínico, y los que tienen buen control glucémico sufren frecuentes episodios de hipoglucemias. Por tanto, es necesario encontrar terapias que simulen lo mejor posible el perfil fisiológico de la insulina de un páncreas normal.

En condiciones normales la célula $\beta$ pancreática consigue un balance adecuado del metabolismo hidrocarbonado mediante un ajuste estrecho entre la secreción de insulina y los niveles de glu- cosa. Se realiza a través de una secreción continua de insulina entre comidas y durante la noche (también denominada secreción basal) y una secreción aguda en respuesta a los alimentos (secreción prandial) 1 . En el momento actual, la mejor estrategia de tratamiento insulínico es el "régimen basal-bolus": los requerimientos basales de insulina a lo largo de 24 horas se cubren con insulina de acción prolongada o por sistemas de infusión continua (bombas de insulina), y las ingestas mediante la administración de insulinas de acción rápida.

\section{Tipos de insulinas}

Los avances en genética molecular (tecnología de ADN recombinante) han permitido sustituir la insulina de origen animal por la insulina humana, y la síntesis de nuevas moléculas dirigidas a un mejor control glucémico. En los últimos años se han ido incorporando al mercado distintos tipos de análogos de insulina (de acción rápida y de acción lenta) cuya respuesta resulta más predecible

Tabla I. Tipos de insulina y tiempos de inicio de acción, pico máximo y duración

\begin{tabular}{lccccc}
\hline Tipo insulina & Regular & NPH & AAR & Glargina & Detemir \\
\hline Inicio acción & $30^{\prime}-45^{\prime}$ & $1-2 \mathrm{~h}$ & $10^{\prime}-15^{\prime}$ & $2-3 \mathrm{~h}$ & $1-2 \mathrm{~h}$ \\
\hline Máximo efecto & $1-3 \mathrm{~h}$ & $3-6 \mathrm{~h}$ & $30^{\prime}-90^{\prime}$ & Poco pico & $5-8 \mathrm{~h}$ \\
Duración & $5-6 \mathrm{~h}$ & $8-12 \mathrm{~h}$ & $3-4 \mathrm{~h}$ & $20-24 \mathrm{~h}$ & $12-20 \mathrm{~h}$ \\
\hline
\end{tabular}

AAR: análogos acción rápida (lispro, aspártico y glulisina). 
que la de sus homólogos (insulina regular e insulina $\mathrm{NPH}$, respectivamente) $)^{2}$.

En el momento actual hay diferentes tipos de insulinas con distintos perfiles de acción que se utilizan de forma asociada para imitar el patrón de secreción fisiológica (tabla I).

\section{Insulinas de acción rápida}

Se utilizan fundamentalmente para el control de las glucemias postprandiales, para corregir la hiperglucemia incidental y para el tratamiento ambulatorio de situaciones con cetosis.

\section{a) Insulina regular}

\section{(Actrapid ${ }^{\circledast}$ o Humulina Regular $\left.{ }^{\circledR}\right)$}

Aunque no remeda la secreción fisiológica de insulina, se puede usar asociada a insulina intermedia o a análogos de acción lenta o en perfusiones de insulina vía intravenosa.

El inicio de su acción es tardío (a la media hora), con un "pico" importante entre 1-2 horas, y una duración prolongada (de 5 a 6 horas). Por este motivo se debe administrar media hora antes de las comidas y es necesario tomar alguna cantidad de hidratos de carbono a las 2-3 horas de su administración para evitar hipoglucemias tardías. Inconvenientes que desaparecen con la utilización de los análogos de acción rápida.

\section{b) Análogos de insulina de acción rápida (AAR)}

La modificación de la estructura molecular de la insulina regular (porción Cterminal de la cadena B) logra características farmacocinéticas diferentes, con un comienzo de acción más rápido y un perfil de actividad más corto, que se atribuye a la reducción de la capacidad de autoasociación para formar hexámeros y a una mayor velocidad de disociación de los mismos en el tejido subcutáneo ${ }^{3}$.

El inicio de acción más rápido de estas insulinas permite su administración justo antes de las comidas, e incluso ha demostrado su eficacia administrada tras las tomas, motivo por el que se utiliza en niños pequeños con ingestas caprichosas ${ }^{4}$.

Su vida media es menor, su acción resulta más predecible y reduce la tasa de hipoglucemias con mejor control de la glucemia postprandial ${ }^{5}$.

En la actualidad disponemos de tres AAR: la insulina lispro (Humalog ${ }^{\circledR}$ ), la insulina aspártico (Novorapid ${ }^{\oplus}$ ) y la insulina glulisina (Apidra ${ }^{\oplus}$ ). El perfil farmacocinético de las tres es similar, aunque en la práctica muestran algunas pequeñas diferencias.

- Insulina lispro (Humalog ${ }^{\circledR}$ ): se obtiene modificando el aminoácido 
prolina de la posición 28 de la cadena B por lisina y la lisina de la posición 29 por prolina. Su acción empieza a los 10-15 minutos, con un pico a los 30-90 y una duración de 3-4 horas.

- Insulina aspártico (Aspart ${ }^{\circledR}$ ): se obtiene por sustitución de la prolina en la posición 28 de la cadena B por un ácido aspártico. Inicia su actividad algo más tarde que la insulina lispro y tiene mayor duración ${ }^{6}$.

- Insulina glulisina (Apidra ${ }^{\oplus}$ ): difiere de la insulina regular en dos aminoácidos, la asparragina de la posición B3 es sustituida por lisina y la lisina de B29 por ácido glutámico. Su acción se inicia algo antes que la insulina lispro ${ }^{7}$. En la actualidad ya está autorizado su uso para mayores de 6 años.

\section{Insulinas de acción retardada}

El mejor control de la glucemia postprandial, obtenido con los AAR, solo se traduce en una mejoría del control metabólico si se asocia a la sustitución adecuada de la insulina basal ${ }^{8}$. La insulina basal ideal sería aquella de larga duración, que no tuviera pico de acción, reproducible y que imitara la secreción de insulina interprandial de los sujetos sanos. En el momento actual como insuli- nas basales se pueden utilizar las insulinas de acción intermedia o los análogos de acción prolongada.

\section{a) Insulina de acción intermedia (NPH)}

Se obtiene tras la adición de protamina a la molécula de la insulina, consiguiendo una curva de acción lenta. Tiene un perfil de actividad con una duración inferior a 12 horas que obliga a administrarla varias veces al día. Además, presenta gran variabilidad de absorción y acción (tanto inter- como intraindividual de hasta un $68 \%)^{9}$ que dificulta en muchas ocasiones su manejo. Por otro lado, su pico de acción pronunciado incrementa el riesgo de hipoglucemias tardías, especialmente durante la noche, y obliga a horarios de ingesta más rigurosos ${ }^{10}$.

\section{b) Análogos de acción prolongada}

Los dos análogos de acción prolongada que existen en la actualidad presentan un perfil de actividad más fisiológico: menor pico de acción y actividad más predecible, y por tanto son mejores sustitutos de la insulina basal. Son la insulina glargina y la insulina detemir.

\section{Insulina glargina $\left(\right.$ Lantus $\left.^{\circledR}\right)$}

Se obtiene mediante técnicas de recombinación genética, al añadir a la insulina humana dos moléculas de argini- 
na en la región C-terminal de la cadena $B$ y sustituir la asparagina por glicina en la posición A21 de la cadena A. Cuando se administra por vía subcutánea forma un microprecipitado al pH del tejido celular subcutáneo, lo cual retrasa su absorción y aumenta su duración, permitiendo un aporte constante de insulina que remeda mejor la secreción basal de insulina fisiológica ${ }^{11}$. El inicio de la actividad es a las 2 horas, la duración de 22 horas con una acción bastante plana y una variabilidad cercana al $48 \%{ }^{7}$. El mayor riesgo de hipoglucemia ocurre a las 6-8 horas de su administración ${ }^{12}$, por eso algunos pacientes se la administran por la mañana o al mediodía, en vez de por la noche, para evitar hipoglucemias nocturnas. La insulina glargina es igual de eficaz administrada a cualquier hora del día ${ }^{13}$. Esta insulina no se puede mezclar con otras insulinas.

\section{Insulina detemir (Levemir ${ }^{\circledR}$ )}

Análogo de insulina soluble obtenido por la unión a la molécula de la insulina de un ácido graso (ácido mirístico) que se liga a los receptores presentes en la albúmina de forma reversible, de manera que enlentece su absorción y se prolonga su acción. Esta insulina se une a la albúmina en un $98 \%$, y solo su fracción libre puede actuar sobre los receptores de insulina de las células diana. Es soluble a pH neutro, por lo que tras su inyección subcutánea permanece líquida $y$, por tanto, con menor variabilidad en su absorción' 7 . Tiene menos potencia hipoglucemiante que la insulina NPH, obtiene mejores niveles de glucemia en ayunas, con menos variabilidad y menor ganancia de peso ${ }^{14}$. En el niño y el adolescente hay que administrarla dos veces al día, ya que su duración de hasta 20 horas solo se alcanza con dosis altas.

\section{Régimen de tratamiento insulínico}

En la DM1 las pautas de insulina deben ser individualizadas y adaptadas a las características y perfil glucémico de cada paciente. La insulinoterapia forma parte del tratamiento global, en el que a través de la educación diabetológica se obtienen los conocimientos necesarios para realizar correctamente cambios en la dieta, el ejercicio y/o la insulina, con el objetivo de mantener glucemias lo más cercanas a la normalidad. Esto exige realizar autoanálisis de glucemia capilar varias veces (media de 5- 6 controles glucémicos al día), para conocer su situación metabólica y poder alcanzar los objetivos del tratamiento. Esta monitorización y autocontrol es uno de los pilares fundamentales del tratamiento. 
La elección del tipo de insulina y el régimen de administración varía en función de: edad del paciente, duración de la diabetes, estilo de vida, capacidad familiar y personal de aprendizaje, cumplimiento terapéutico y cobertura, y capacitación de la unidad de diabetes pediátrica. En la edad pediátrica es necesario con frecuencia cambiar de un régimen insulínico a otro para conseguir los objetivos terapéuticos deseados.

\section{Objetivos terapéuticos}

Los valores de glucemia recomendados en los niños con DM1 son los siguientes:

- En ayunas y antes de cualquier comida: entre 80 y $140 \mathrm{mg} / \mathrm{dl}$.

- 2 horas después de cualquier comida: entre 100 y $180 \mathrm{mg} / \mathrm{dl}$.

- A las 3 de la madrugada: por encima de $80 \mathrm{mg} / \mathrm{dl}$.

Cualquier cifra que se encuentre por encima de estos valores se considera hiperglucemia, y cualquier cifra por debajo de $70 \mathrm{mg} / \mathrm{dl}$, se considera hipoglucemia. Hoy día, con los sistemas de infusión continua de insulina subcutánea, los objetivos glucémicos tienden a ser más bajos, intentando ser más parecidos a la normalidad. En cualquier caso, estos objetivos deben ser individualizados, evitando sobre todo las hi- poglucemias en los niños más pequeños.

\section{Dosis de insulina}

La dosis inicial de insulina depende de la edad al diagnóstico, del estadio puberal y de la presencia o no de cetosis o cetoacidosis al debut de la enfermedad.

En caso de cetoacidosis la insulina recomendada es la regular en perfusión intravenosa continua a 0,05-0,1 Ul/kg/hora. Si existe cetosis sin acidosis puede iniciarse, bien con este tratamiento, o con insulina regular o con AAR por vía subcutánea, entre 0,1 y 0,3 UI/ $/ \mathrm{gg} /$ dosis. En caso de hiperglucemia aislada sin cetosis puede valorarse el inicio con pauta basal-bolus, siendo las necesidades de insulina menores que si existe cetosis.

En general, las necesidades diarias de insulina son las siguientes:

- Fase de remisión parcial o "luna de miel": < 0,5 UI/kg/día.

- Niño prepúber: 0,5-0,7 Ul/kg/día.

- Durante la pubertad: hasta 1-1,5 UI/kg/día.

- Postpúber: 0,7-0,8 UI/kg/día.

Una vez conseguido el ajuste inicial, la dosis diaria de insulina se repartirá en un $40-60 \%$ como insulina basal, bien con análogos de acción prolongada (glargina o detemir) o con insulina intermedia, y el resto como insulina prandial 
con insulina de acción rápida (regular o AAR) antes de cada comida. Las dosis se ajustan a las glucemias pre- y postprandiales y nocturnas.

En algunas etapas de la vida, como en la pubertad, se precisan dosis de insulina más elevadas para conseguir un buen control metabólico, debido a la insulinorresistencia que existe en esta etapa del crecimiento.

\section{Pautas de insulinoterapia}

Las pautas más habituales en Pediatría son las que combinan insulina de acción intermedia o análogos de acción prolongada, con insulina regular o AAR de 3 a 5 dosis diarias.

\section{Pautas con insulina intermedia}

La insulina NPH se sigue utilizando sobre todo en menores de 6 años en los que, en la actualidad, no está recomendado el uso de análogos de acción prolongada.

\section{a) Pauta de 2 dosis al día}

Se puede utilizar solo en fases muy iniciales de la diabetes en el niño prepuberal con secreción residual de insulina. Con 2 dosis de insulina intermedia y regular o AAR antes del desayuno y antes de la cena o merienda. El reparto de la dosis es aproximadamente $2 / 3$ por la mañana y $1 / 3$ en la cena/merienda (el $70 \%$ suele ser $\mathrm{NPH}$ y el $30 \%$ de rápida).

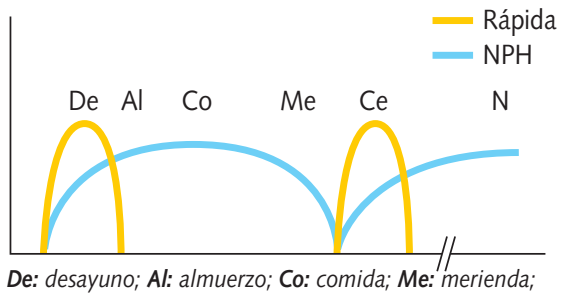
Ce: cena; $N$ : noche.

\section{b) Pauta de 3 dosis al día}

Se administra NPH e insulina regular o AAR antes del desayuno, comida y cena. Las dosis de insulina intermedia deben estar separadas al menos 6-7 horas.

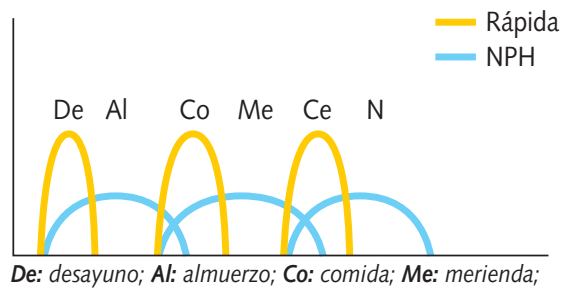
Ce: cena; $N$ : noche.

Cuando el tiempo entre comidas es inferior a 6 horas puede ser suficiente la administración de insulina regular. Por ejemplo, entre el desayuno y la comida en el niño pequeño.

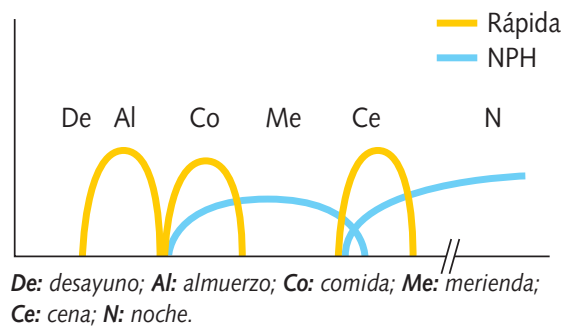




\section{c) Pauta de 4 dosis al día}

Se desdobla la insulina de la cena en rápida (regular o AAR) antes de la cena e insulina NPH 2-3 horas después. Hasta la aparición de los análogos de acción prolongada ha sido la pauta más utilizada durante la pubertad.

En los niños pequeños se puede conseguir un buen control con insulina regular antes del desayuno, la comida y la cena, no separadas más de 5 horas, e insulina NPH 2 ó 3 horas después de la cena.

\section{Pautas con análogos de insulina de acción prolongada}

Son una buena opción para el aporte de la insulinemia basal por su mayor duración, su menor variabilidad inter- e intraindividual y menor pico máximo de acción. Reducen el riesgo de hipoglucemias, sobre todo nocturnas. En los niños se utilizan siempre asociadas a 3-4 inyecciones de insulina de acción rápida (generalmente AAR) para cubrir las diferentes ingestas a lo largo del día (so-

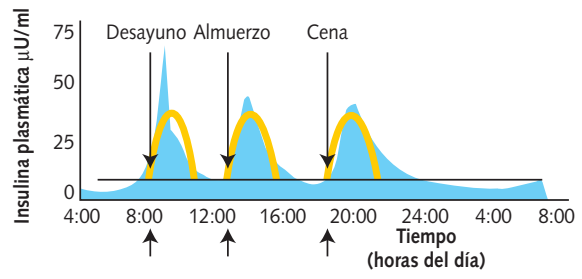

bre todo desayuno, comida, merienda y cena).

\section{a) Pauta con insulina glargina}

Se recomienda administrar insulina Lantus ${ }^{\circledast}$ subcutánea una vez al día, normalmente al irse a la cama. En caso de hipoglucemias nocturnas, que no sean por exceso de dosis, se puede cambiar su administración a la comida o el desayuno'.

Para disminuir el riesgo de hipoglucemia cuando se cambia de una pauta con insulina NPH a Lantus ${ }^{\circledast}$ la dosis inicial de insulina glargina será el $80 \%$ de las unidades aportadas como insulina $\mathrm{NPH}$.

La insulina glargina no se puede mezclar con las restantes insulinas por su pH ácido, lo que supone un total de 4-6 inyecciones de insulina al día. Este problema se contrarresta con una clara ventaja de libertad de horario, dado que los AAR coinciden con las comidas, pero puede llevar a la omisión de alguna dosis de insulina rápida, sobre todo en la adolescencia, con empeoramiento del control metabólico.

\section{b) Pauta con insulina detemir}

Se inicia con 2 dosis de insulina Levemir $^{\circledR}$ al día: ajustadas a las necesidades basales diurnas y nocturnas, o en 2 do- 
sis iguales antes del desayuno y la cena. Además hay que aportar AAR preingesta, como se ha comentado con Lantus ${ }^{\circledast}$.

A veces, en niños impúberes, con los análogos de acción lenta se puede utilizar insulina regular en vez del AAR, sobre todo en la comida, si no se quieren pinchar en la merienda o en el desayuno, si quieren tomar hidratos de carbono a media mañana.

Para cubrir el fenómeno del atardecer (aumento de las necesidades de insulina por la tarde) generalmente es necesario administrar un AAR en la merienda, aunque el niño o el adolescente no meriende.

\section{Terapia con bombas de infusión continua de insulina (ISCI)}

Es un sistema que libera insulina de manera continua en el tejido celular subcutáneo logrando una insulinemia basal; el sistema además libera insulina de forma intermitente ("bolus") previamente a las comidas para evitar la hiperglucemia postprandial, o para corregir las hiperglucemias accidentales. Solo se utilizan insulinas AAR.

La bomba es programada por el paciente o sus padres, después de una intensa educación diabetológica, para aportar: las necesidades basales de insulina ajustadas a las necesidades indivi- duales de cada sujeto, y los bolus de insulina, activados por el propio paciente, para cubrir las comidas y corregir las hiperglucemias ocasionales.

Este tratamiento es una alternativa en pacientes motivados que no logran un buen control glucémico a pesar de múltiples dosis de insulina subcutánea, en diabéticos con hipoglucemias graves o con un importante "fenómeno de alba", o en ocasiones para mejorar la calidad de vida.

Los menores de 10 años necesitan la supervisión continua de un adulto.

\section{Aportes extraordinarios de insulina: índice de sensibilidad}

Para corregir las hiperglucemias accidentales se recomienda usar AAR, aunque puede también utilizarse insulina regular.

El índice o factor de sensibilidad indica cuánto disminuye la glucemia (en $\mathrm{mg} / \mathrm{dl}$ ) una unidad de insulina de acción rápida. Se calcula mediante un cociente en cuyo numerador figura un número fijo (1.800 si se utiliza AAR y 1.500 si es insulina regular) y el denominador es el número total de unidades de insulina al día que el niño necesita.

Índice de sensibilidad $=1.800$ / dosis total de insulina al día (con AAR) 
Hay que tener en cuenta que cuando el paciente presenta cetosis las necesidades de insulina pueden ser mayores.

La dosis de insulina correctora (número de unidades de AAR a administrar en caso de hiperglucemia, aproximadamente cada 3 horas) se calcula mediante el cociente:

Glucemia actual - glucemia ideal / índice de sensibilidad

\section{Conclusiones}

Los análogos de insulina han aumentado el abanico de posibilidades para diseñar estrategias de insulinoterapia adap- tadas a la edad y el estilo de vida de cada niño y adolescente con diabetes, para conseguir los objetivos de tratamiento propuestos.

Sin embargo, el manejo de la diabetes no es solo un régimen insulínico. Precisa de un equipo multidisciplinario de atención diabetológica pediátrica que, mediante la educación diabetológica, instruya al niño o al adolescente y a su entorno a manejar los pilares básicos del tratamiento (insulina, dieta y ejercicio a través de un correcto autocontrol), tanto en su vida cotidiana como en aquellas situaciones extraordinarias que requieran ajustes del mismo.

\section{Bibliografía}

1. Owens DR, Zinman B, Bolli GB. Insulins today and beyond. Lancet. 2001;358:739-46.

2. Brange J, Volund A. Insulin analogs with improved pharmacokinetic profiles. Adv Drug Delivery Rev. 1999;35:307-35.

3. Bolli GB, Di Marchi RD, Park GD, Pramming $S$, Koivisto VA. Insulin analogs and their potential in the management of diabetes mellitus. Diabetologia. 1999;42:1151-67.

4. Schernthaner G, Wein W, Sandholzer K, Equiluz-Bruck S, Bates PC, Birkett MA. Postprandial insulin lispro. A new therapeutic option for type 1 diabetic patients. Diabetes Care. 1998;21:570-3.

5. Dahl-Jorgensen K. Modern insulin therapy in children and adolescents. Acta Paediatr Suppl. 1999;427:25-30.
6. Barrio R, Gussinyé $M$, Hermoso F, González I Gómez A, López MJ, y col. Insulinoterapia 2006. Tratamiento insulínico en el niño y el adolescente. Comisión de Diabetes Infantil de la Sociedad Española de Endocrinología Pediátrica.

7. Heise $T$, Nosek L, Spitzer $H$, Heinemann L, Niemöller $E$, Frick AD, Becker RHA. Insulin glulisine: a faster onset of action compared with insulin lispro Diabetes Obes Metab. 2007;9:746-53.

8. Ebeling P, Jansson PA, Smith U, Lalli C, Bolli $G B$, Koivisto VA. Strategies toward improved control during insulin Lispro therapy in IDDM: importance of basal insulin. Diabetes Care. 1997;20: 1287-9.

9. Heise U, Nosek L, Biilmann RB, Endahl L, Heinemann L, Kapitza C, Draeger E. Lower withinsubject variability of insulin detemir in comparison 
to NPH insulin and insulin glargine in people with type 1 diabetes. Diabetes. 2004;53:1614-20.

10. Lepore M, Pampanelli S, Fanelli C, Porcellati F, Bartocci L, Di Vicenzo A, y col. Pharmacokinetics and pharmacodynamics of subcutaneous injection of long-acting human insulin analog glargine, NPH insulin, and ultralente human insulin and continuous subcutaneous infusion of insulin lispro. Diabetes. 2000;49:2142-8.

11. Levien $T L$, Baker DE, White JR, Campbell RK. Insulin glargine: a new basal insulin. Ann Pharmacother. 2002;36:1019-27.

12. Plank J, Bodenlenz $M$, Sinner $F$, Magnes $C$, Görzer E, Regittnig W, et al. A double-blind randomized, dose-response study investigating the pharmacodynamic and pharmacokinetic properties of the long-acting insulin analog detemir. Diabetes Care. 2005;28:1107-2.

13. Hamann A, Matthaei S, Rosak Ch, Silvestre L for the HOE901/4007 Study Group. A randomized clinical trial comparing breakfast, dinner, or bedtime administration of insulin glargine in patients with type 1 diabetes. Diabetes Care. 2003; 26:1738-44.

14. Heise U, Nosek L, Biilmann RB, Endahl L, Heinemann L, Kapitza C, Draeger E. Lower within-subject variability of insulin detemir in comparison to NPH insulin and insulin glargine in people with type 1 diabetes. Diabetes. 2004;53: 1614-20. 